\title{
Solvent-Induced Acceleration of the Rate of Activation of a Molecular Reaction
}

\author{
P. L. García-Müller and F. Borondo \\ Departamento Química and Instituto Mixto de Ciencias Matemáticas CSIC-UAM-UC3M-UCM, Universidad Autónoma de Madrid, \\ Cantoblanco, 28049 Madrid, Spain \\ Rigoberto Hernandez \\ Center for Computational Molecular Sciences and Technology, School of Chemistry and Biochemistry, \\ and Georgia Institute of Technology, Atlanta, Georgia 30332-0430, USA \\ R. M. Benito \\ Grupo de Sistemas Complejos, and Departamento de Física y Mecánica, Escuela Técnica Superior de Ingenieros Agrónomos, \\ Universidad Politécnica de Madrid, 28040 Madrid, Spain
}

\begin{abstract}
An increase in the rates of activated processes with the coupling to the solvent has long been predicted through the phenomenological Langevin equation in the weak coupling regime. However, its direct observation in particle-based models has been elusive because the coupling typically places the processes in the spacial-diffusion limited regime wherein rates decrease with increasing friction. In this work, the forward and backward reaction rates of the $\mathrm{LiNC} \rightleftharpoons \mathrm{LiCN}$ isomerization reaction in a bath of argon atoms at various densities have been calculated directly using molecular dynamics trajectories. The so-called Kramers turnover in the rate with microscopic friction is clearly visible, thus providing direct and unambiguous evidence for the energy-diffusion regime in which rates increase with friction.
\end{abstract}

The activation of a quasibound species above its trapping potential is the rate determining step in many processes, and it is certainly central in chemical reactions The interaction between the reactants with the solvent (or other degrees of freedom) must transfer sufficient energy to activate the reactants above the energy barrier leading to products. The corresponding rate should therefore increase with the coupling represented by friction. An increase in friction, however, also slows down the reactants and induces a competing mechanism that reduces the rate. This turnover, from an energy-diffusion limited regime, where rates increase with friction, to a spacial-diffusion one, in which rates do the opposite, was predicted by Kramers in his 1940 seminal paper on reaction-rate theory. Moreover, the entire regime lies below the transition state theory estimate which is another breakthrough in molecular dynamics that appeared in the literature about the same time as Kramers' work . This simple treatment of reaction rates assumes that the reactants always maintain a thermal distribution and that, once activated, always react. This upper bound to the true reaction rate has been improved by recent advances in the representation of the interaction region, and in how it is connected to the global surface when reactions are solvated in liquids

The open question to be addressed here is whether the energy-diffusion regime within the Kramers turnover is realizable and hence relevant to chemical dynamics, let alone other physical processes. Abrash et al. and Nikowa et al. have independently seen a turnover in the isomerization of trans-stilbene, though the origin of the behavior could not be definitively associated with friction. Ashcroft and co-workers observed such behavior in the isomerization of cyclohexane and in the internal rotation rate of $N, N$-dimethyltrichloroacetamide with decreasing pressure. Meanwhile, significant theoretical and computational models have been developed to describe the Kramers turnover . The theory has been applied to many model systems within the framework of reduced-dimensional Langevin equations, e.g., isomerization reactions and surface diffusion . The critical missing piece in this story has been the clear observation of the Kramers turnover in a molecular isomerization taking place in a molecular solvent from the low to high density regime in which each of the components has been mapped directly to the turnover theory. A study of the $\mathrm{CH}_{3}-\mathrm{CN} \rightleftharpoons \mathrm{CN}-\mathrm{CH}_{3}$ isomerization was suggestive, but not definitive, because it employed a very crude solvation model in which only eight argon atoms comprised the solvent. The present work provides definitive evidence of the Kramers turnover in the $\mathrm{LiNC} \rightleftharpoons \mathrm{LiCN}$ isomerization rates as the density of the argon solvent is varied.

Classical molecular dynamics simulations, allowing for the calculation of the forward and backward reaction rates for the isomerization of $\mathrm{LiCN}$ in argon solvents, have been performed at various densities. As described within the text, the pairwise additive potentials have all been fully vetted in past work, and hence provide a reasonable representation of the actual dynamics. Additional molecular 
dynamics simulations have also been performed to obtain the potential of mean force and friction along the reaction coordinate for the isomerization. The latter allows us to relate the argon density directly to the friction, and in turn plot the observed rate as a function of microscopic friction. The observed Kramers turnover can be obtained quantitatively in this picture through the Pollak-Grabert-Hänggi (PGH) turnover formula which yields the rates across the reduced-dimensional Langevin model for this reaction. The remarkable agreement between the Kramers turnover theory and the numerical simulations thus verifies the possibility for reaction rates to be dominated by energy-diffusion limitations on the reactants when the reaction takes place in the presence of sufficiently low density solvents.

Lithium cyanide, $\mathrm{LiCN}$, is a triatomic molecule with three internal degrees of freedom. It can be described by Jacobi coordinates: the distance $R$ between the $\mathrm{Li}$ atom and the center of mass of the $\mathrm{C} \equiv \mathrm{N}$ bond, the interatomic distance $r$ of the $\mathrm{CN}$ bond, and the bending angle $\psi$ between the $\mathrm{C} \equiv \mathrm{N}$ bond axis and the direction spanned by $R$. The interatomic distance $r$ is typically frozen at its equilibrium values because the $\mathrm{C} \equiv \mathrm{N}$ vibrational frequency is much higher than that of the other modes. The internal dynamics thus reduces to the 2 degrees of freedom described by the coordinates $R$ and $\psi$. The potential energy surface, $V_{\text {int }}^{\mathrm{LiCN}}$, in these two variables has been fit to $a b$ initio electronic structure calculations data by Essers et al [23]. Its contour plot is shown at the left side of Fig. 1. It contains two potential wells corresponding to linear isomers, $\mathrm{LiCN}$ at $\psi=0$ and $\mathrm{LiNC}$ at $\psi=\pi$, separated by a modest barrier of just 0.0159 a.u. $(=5020 \mathrm{~K})$ in the direction of the forward reaction, $\mathrm{LiNC} \rightarrow \mathrm{LiCN}$, and 0.0050 a.u. $(=1600 \mathrm{~K})$ in the backward direction. The minimum energy path (MEP) connecting the two isomer
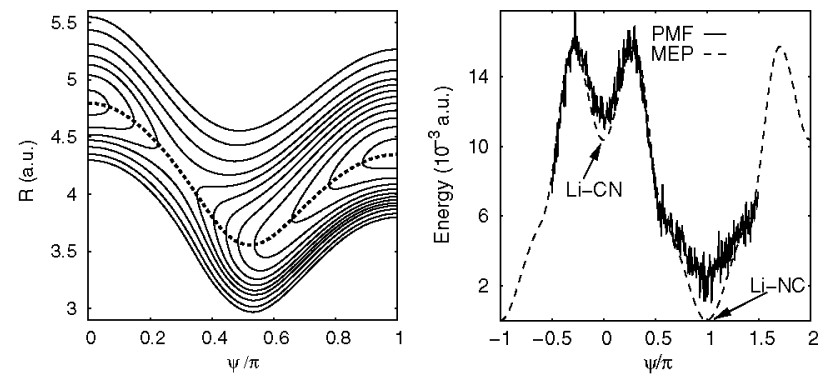

FIG. 1. Left: Contour plot of the internal potential of the $\mathrm{LiNC} / \mathrm{LiCN}$ molecular system in terms of the Jacobi coordinates with distance $R$ and angle $\psi$ of the Li relative to the center of mass of $\mathrm{CN}$ and its bond axis. The dashed curve highlights the minimum energy path that connects the two isomer wells. Right: The potential of the mean force $V_{\mathrm{PMF}}$ computed from Eq. (2) assuming a bath thermal energy of $k_{B} T=0.01$ a.u. (full line), and the energy profile of the SBB model for the bare $\mathrm{LiCN} / \mathrm{LiNC}$ molecular system (dashed line) along the minimum energy path. wells is highlighted on the contour plot shown at the left of Fig. 1, and its energy profile is shown at the right.

The forward and backward isomerization reactions have been simulated by classical molecular dynamics at the microscopic level. The LiNC molecule has been embedded in a bath of Ar atoms whose average interatomic distances range from 5 to 30 a.u. depending on the specific density. The total potential energy is

$$
V=\sum_{i}^{N} \sum_{i>j}^{N} V_{i j}^{\mathrm{Ar}-\mathrm{Ar}}+V_{\mathrm{int}}^{\mathrm{LiCN}}+\sum_{j}^{N}\left(V_{j}^{\mathrm{Ar}-\mathrm{Li}}+V_{j}^{\mathrm{Ar}-\mathrm{C}}+V_{j}^{\mathrm{Ar}-\mathrm{N}}\right),
$$

where the superscript, $\operatorname{Ar}-X$, indicates the different twobody interactions, and $V_{\mathrm{int}}^{\mathrm{LiCN}}$ is the internal molecular potential. All of the two-center potentials between nonbonded atoms were taken to be two-body Lennard-Jones short-range potentials using standard parametrizations.

The propagation of the molecular dynamics has been performed using in-house codes capable of integrating Lagrange equations of motion for all the atoms using the Verlet algorithm, periodic boundary conditions, and interaction lists. A set of $512 \mathrm{Ar}$ atoms has been included in the periodic box representing the bath at a temperature of $3500 \mathrm{~K}$. At this temperature, the ratio of thermal energies to the barrier height, $V^{+} / k_{B} T$, is 1.434 and 0.44 for the forward and backward reaction, respectively. The temperature is established during the initialization phase wherein the Ar velocities are rescaled at each step so that the overall average kinetic energy matches the equilibrium temperature. Thereafter, during the calculation phase, the system is propagated without the use of any thermostat or temperature rescaling.

The reaction rates have been calculated using rate equations based on the flux of reactive trajectories through a dividing surface along the reaction coordinate (i.e., the bending of the $\mathrm{Li}-\mathrm{CN}$ bond). Trajectories are considered to be reactive if they leave the reactant well, cross the energy barrier, and arrive at the product well. Such trajectories contribute only once to the flux, regardless of the number of barrier recrossings they encounter, and hence this calculation is formally exact. The forward $k_{f}$ and backward $k_{b}$ reaction rates have been calculated at several bath densities, ranging from 0.5 to $70 \mathrm{~mol} / 1$. Their ratio, the equilibrium constant $K$, has been evaluated at several temperatures to validate microscopic reversibility-viz. that $K$ scales exponentially with the inverse of the temperature of the system as $K=e^{-\beta \Delta G}$, were $\Delta G$ is the Gibbs free energy difference between product and reactant equilibria. The bath friction kernel has also been calculated from the autocorrelation of the forces exerted on the $\mathrm{LiCN}$ center of mass. The bath viscosity is the zero frequency component of the Laplace transform of the bath friction kernel. Hence, the values calculated for these rates can be compared directly to the PGH theory predictions.

The reduced-dimensional calculations require a potential of mean force (PMF). This has been determined ap- 
TABLE I. Parameters defining the effective one-dimensional Straub-Borkovec-Berne potential in the Langevin model for the $\mathrm{LiNC} \rightleftharpoons \mathrm{LiCN}$ isomerization reaction. All quantities listed are in atomic units when not dimensionless.

\begin{tabular}{|c|c|c|c|c|c|}
\hline $\mathrm{LiNC} \rightarrow \mathrm{LiCN}$ & $V^{+}$ & $\psi_{0}(\mathrm{rad})$ & $\omega^{+}$ & $\omega_{\mathrm{LiNC}}$ & $\alpha^{*}$ \\
\hline MEP & 0.016 & 2.23 & $8.0 \times 10^{-4}$ & $4.4 \times 10^{-4}$ & 0.84 \\
\hline PMF & 0.013 & 2.32 & $8.0 \times 10^{-4}$ & $3.6 \times 10^{-4}$ & 0.84 \\
\hline $\mathrm{LiCN} \rightarrow \mathrm{LiNC}$ & $V^{+}$ & $\psi_{0}(\mathrm{rad})$ & $\omega^{+}$ & $\omega_{\mathrm{LiCN}}$ & $\alpha^{*}$ \\
\hline MEP & 0.005 & 0.91 & $8.0 \times 10^{-4}$ & $6.9 \times 10^{-4}$ & 0.84 \\
\hline PMF & 0.004 & 0.82 & $8.0 \times 10^{-4}$ & $6.8 \times 10^{-4}$ & 0.84 \\
\hline
\end{tabular}

proximately using the form of the Straub-Borkovec-Berne (SBB) potential model with parameters fitted to the corresponding simulated potential. As the SBB model is locally harmonic, it does not include any local anharmonicities. In the SBB model, the LiCN isomer well, the barrier, and the LiNC isomer well are approximated by continuous piecewise parabolic potentials, which are defined in terms of the barrier height relative to the potential wells $V^{+}$, the equilibrium position of the two isomers with respect to the equilibrium position of the barrier $\psi_{0}$, the barrier frequency $\omega^{+}$, and the frequencies at the minima of the potential wells $\omega_{\mathrm{LiNC}}$ and $\omega_{\mathrm{LiCN}}$. Values for the parameters corresponding to the bare (viz. MEP) and the PMF are summarized in Table I for the forward and backward reactions. The fit of the $\mathrm{SBB}$ model to the bare potential along the MEP connecting both isomer wells is shown at the right in Fig. 1. The PMF is also displayed in this figure as a solid line, and has been determined from the Boltzmann weighted average

$$
V_{\mathrm{PMF}}(\psi)=-\frac{1}{\beta} \log \left\{\int d q \delta\left[\psi^{\prime}(q)-\psi\right] e^{-\beta V(q)}\right\},
$$

where $q$ denotes all the Cartesian coordinates of all the atoms in the system, $V$ is the interaction potential described in Eq. (1), $\psi$ is the reaction coordinate, and $\beta^{-1}=$ $k_{B} T$. The memory in the PGH model has been surmised through an exponential friction kernel $\gamma(t)$, and its Laplace transform:

$$
\begin{gathered}
\gamma(t)=\frac{e^{-t / \alpha \gamma}}{\alpha} \\
\hat{\gamma}(s)=\frac{\gamma}{1+s \gamma \alpha},
\end{gathered}
$$

where $\gamma$ is the damping parameter and $\alpha$ is the inverse of the infinite frequency shear modulus of the solvent. The latter parameter has been adjusted to obtain a best fit to the memory function calculated by molecular dynamics simulations. The PGH rates have been calculated at each set of conditions with respect to the PMF and the bare potential as a reference. As the flux across the barrier in the PMF accounts for only one of the two symmetrically identical open channels to reaction, the PGH rates must be multiplied by a factor two before comparison with the direct numerical simulations. As can be seen in Fig. 2, the Laplace transform of the friction memory function is reasonably well approximated by Eq. (4).

The reaction rates obtained by molecular dynamics simulations are shown in Fig. 3 and compared to the PGH model predictions. The forward reaction rates are higher than the backward rates as one would expect due to the different energy values of the barriers. The reaction rates increase with the bath friction in the energy-diffusion regime $\left(\gamma^{*}<0.2\right)$ until they reach the Kramers turnover. This effect is clearly visible in both reactions though they evidently occur at different $\gamma^{*}\left(=\gamma / \omega^{+}\right)$values. The reaction rates start to decline with the further increase of the bath viscosity (density) as is expected in the spatial diffusion regime $\left(\gamma^{*}>0.5\right)$.

The PGH theory predictions are in good agreement with the molecular dynamics simulations. Both the PMF and the MEP potentials have been addressed, giving no significant differences. This counterintuitive result is likely due to insufficient averaging. Nevertheless, the key point here is that the role of friction in the turnover is in nearly direct agreement between the numerical simulations and the reduced-dimensional models. In order to achieve convergence in this study, a relatively high temperature has been employed. It is well above the range for which the PGH formula was shown to be applicable, and yet the agreement is remarkably good. This is likely a result of the fact that the reactant well is sufficiently broad as to admit a steady-

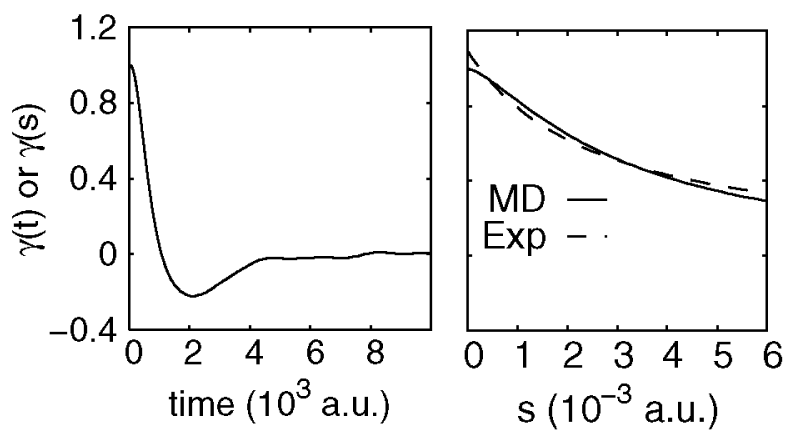

FIG. 2. The friction memory function, displayed at left, has been obtained from the autocorrelation of the forces exerted by the bath (at a density of $45.5 \mathrm{~mol} / \mathrm{l}$ ) on the molecular center of mass. The Laplace transform of this numerical memory function is shown as a solid curve in the figure on the right. Therein, the dashed line represents the Laplace transform of the best-fitted exponential memory kernel. 


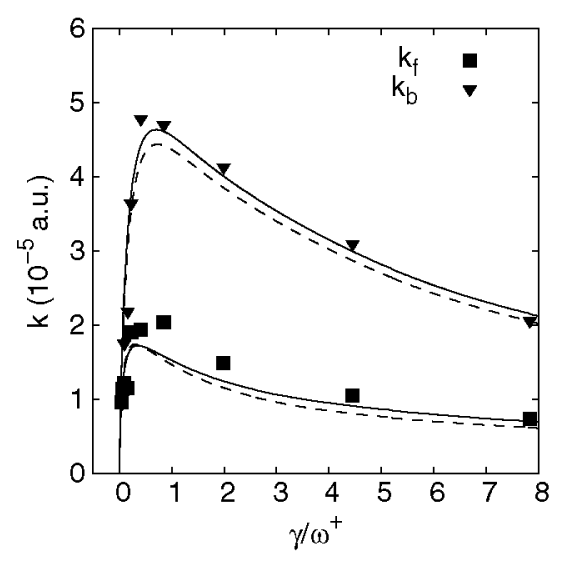

FIG. 3. The reaction rate is shown as a function of the bath viscosity normalized to the barrier frequency for a bath thermal energy equal to 0.0110839 a.u. $(=3500 \mathrm{~K})$. The forward reaction rates $k_{f}$ (squares) and backward reaction rates $k_{b}$ (inverted triangle) have been calculated directly using molecular dynamics simulations. The curves correspond to the Pollak-GrabertHänggi theory predictions for the bare MEP (solid lines) and the PMF potential (dashed lines).

state rate, but this conjecture is open for future debate. Meanwhile, lower temperatures could be accessed through the use of rate formulas (for the direct dynamics) that would need to offer faster convergence without requiring substantially more computer resources. In this direction, it may be possible to apply accelerated dynamics , transition path sampling , or the moving transition state approach . The success of this model chemical system at reproducing the Kramers turnover also makes one hopeful that it might reveal the extent to which the inclusion of stochastic acceleration is important in the choice of coordinate system

In summary, the $\mathrm{LiNC} \rightleftharpoons \mathrm{LiCN}$ isomerization reaction rates in a bath of argon atoms have been calculated using classical molecular dynamics simulations. By varying the density, the interaction between the bath and the isomerization reaction path has been varied across the energy diffusion, Kramers turnover, and spatial diffusion regimes. The observed forward and backward reaction rates have exhibited the expected qualitative turnover across these regimes, and agrees quantitatively with the rates in reduced-dimensional Langevin models. The latter are obtained using the PGH theory and evaluated for a onedimensional potential (viz. a continuous piecewiseparabolic approximation to the MEP) and a memory represented by an exponential friction kernel.

This work has been partly supported by the Spanish MEC under Projects Ingenio Mathematica (i-MATH) No. CSD2006-00032 and No. MTM2006-15533, the Comunidad de Madrid (SIMUMAT S0505/ESP0158), the U.S. National Science Foundation (CHE 0749580), and by the Alexander von Humboldt Foundation. We thank Professor Eli Pollak and Professor David Farrelly for a critical reading of this manuscript. We also thank the late Professor Lorenzo Pueyo for many fruitful discussions.

E. Pollak and P. Talkner, Chaos 15, 026116 (2005).

H. A. Kramers, Physica (Utrecht) 7, 284 (1940).

P. Hänggi, P. Talkner, and M. Borkovec, Rev. Mod. Phys. 62, 251 (1990), and references therein.

H. Eyring, J. Chem. Phys. 3, 107 (1935); E. Wigner, Trans. Faraday Soc. 34, 29 (1938).

S. Wiggins, L. Wiesenfeld, C. Jaffe, and T. Uzer, Phys. Rev. Lett. 86, 5478 (2001).

E. Vanden-Eijnden and F. A. Tal, J. Chem. Phys. 123, 184103 (2005).

H. Waalkens, A. Burbanks, and S. Wiggins, Phys. Rev. Lett. 95, 084301 (2005).

G. Henkelman and H. Jonsson, Phys. Rev. Lett. 90, 116101 (2003).

E. Beerdsen, B. Smit, and D. Dubbeldam, Phys. Rev. Lett. 93, 248301 (2004).

T. Bartsch, R. Hernandez, and T. Uzer, Phys. Rev. Lett. 95, 058301 (2005).

T. Bartsch, T. Uzer, J. M. Moix, and R. Hernandez, J. Phys. Chem. B 112, 206 (2008).

T. Bartsch, J. M. Moix, R. Hernandez, S. Kawai, and T. Uzer, in Advances in Chemical Physics, edited by Stuart A. Rice (John Wiley \& Sons, Hoboken, NJ, 2008), Vol. 140, p. 191.

S. Abrash, S. Repinec, and R. M. Hochstrasser, J. Chem. Phys. 93, 1041 (1990).

L. Nikowa, D. Schwarzer, and J. Troe, J. Chem. Phys. 97, 4827 (1992).

J. Ashcroft, C.-L. Xie, and J. Jonas, J. Chem. Phys. 90, 5386 (1989).

C.-L. Xie, D. Campbell, and J. Jonas, J. Chem. Phys. 92, 3736 (1990).

V. I. Mel'nikov and S. V. Meshkov, J. Chem. Phys. 85, 1018 (1986).

E. Pollak, H. Grabert, and P. Hanggi, J. Chem. Phys. 91, 4073 (1989).

G. Gershinksy and E. Pollak, J. Chem. Phys. 108, 9186 (1998).

S. Miret-Artés and E. Pollak, J. Phys. Condens. Matter 17, S4133 (2005).

J. M. Moix, T. D. Shepherd, and R. Hernandez, J. Phys. Chem. B 108, 19476 (2004).

A. J. Marks, J. N. Murrel, and A.J. Stace, Chem. Phys. Lett. 154, 492 (1989).

R. Essers, J. Tennyson, and P.E. S. Wormer, Chem. Phys. Lett. 89, 223 (1982).

J. E. Straub, M. Borkovec, and B. J. Berne, J. Phys. Chem. 83, 3172 (1985).

A. F. Voter, F. Montalenti, and T. C. Germann, Annu. Rev. Mater. Res. 32, 321 (2002).

P. G. Bolhuis, D. Chandler, C. Dellago, and P. Geissler, Annu. Rev. Phys. Chem. 53, 291 (2002).

J. M. Moix, R. Hernandez, and E. Pollak, J. Phys. Chem. B 112, 213 (2008). 